\title{
Aplicación de la teoría de restricciones en la implementación de un Sistema de Manufactura CAD-CAM en la industria Metalmecánica- Plástica
}

\section{(The theory of constraints applied in a manufacture CAD-CAM system in the industry Metalworking-plastic)}

\author{
Luis Juiña ${ }^{1}$, Víctor Hugo Cabrera ${ }^{1}$, Salvatore Reina²
}

\begin{abstract}
Resumen:
En el presente proyecto se aplica la teoría de restricciones para implementar un sistema de manufactura CAD-CAM en la industria del sector metalmecánico, en productos plásticos, mediante procesos de inyección y soplado de polímeros. En la investigación se determinó que la fabricación del molde para el producto a evaluar tuvo una duración de 223,17 horas y para el procesamiento de $240 \mathrm{~kg}$ de materia prima fue de 25 horas. En el flujo de operaciones para la fabricación del molde se encontró restricción en el servicio contratado de manufactura CNC con 120 horas de duración que corresponde al $51,47 \%$ del tiempo total de elaboración. La fase de diseño, tomó 60 horas que corresponde al $26,88 \%$ del tiempo total del proyecto. Para reducir los tiempos se mejoró el proceso de diseño y manufactura, mediante la implementación de un sistema moderno de diseño CAD y un proceso de fabricación CAM. Para elevar la restricción se cambia de un entorno 2D de trabajo a un sistema de diseño 3D, y se invirtió en una máquina con tecnología CNC. Con la implementación del sistema el tiempo se redujo en un $79 \%$ en el diseño, con respecto al proceso manufactura CNC el tiempo se redujo en un $88 \%$.
\end{abstract}

Palabras clave: teoría de restricciones (TOC); polímero; control numérico computarizado (CNC); CAD; CAM.

\begin{abstract}
:
In the following project, the theory of constraints was applied in order to implement a manufacture CAD-CAM system into the metal mechanic industry processes of polymers injection and blown of polymers. The research showed that the manufacture of the mold with the engraving took 223,17 hours. In the workflow for the manufacture of the mold, a restriction was found in the outsource service of CNC. It took 120 hours of the whole process and represent the 51,47\% the total time of tooling manufacturing. There is also a constraint found in the design time. It was 60 hours that corresponds to $26,88 \%$ of the overall time. In order to reduce the time, a modern system of design in $3 \mathrm{D}$ and CAM was established to improve the model process of design and manufacture. A simulation by computational resource was applied to the plastic. The design was changed from $2 \mathrm{D}$ to 3D. The implementation was focused in the design. A software was installed to improve the speed of modeling methods with reliable information. In the manufacture of molds, a new CNC machine was acquired with three simultaneous axes to eliminate the outsource service. By acquiring the design system, the working time was diminished in $79 \%$ and regarding to the CNC process, the working time was improved in $88 \%$.
\end{abstract}

Keywords: theory of constrains; polymer; computerized numerical control; CAD; CAM.

\footnotetext{
1 Universidad Politécnica Salesiana, Quito - Ecuador ( \{ljuina, vcabrera\} @ups.edu.ec )

${ }^{2}$ Escuela Politécnica Nacional, Quito - Ecuador (salvatore.reina@epn.edu.ec)
} 


\section{Introducción}

Actualmente el país está en un proceso de fortalecimiento de la industria local, por lo cual el Gobierno Nacional plantea transformar el patrón de especialización de la economía ecuatoriana logrando una inserción estratégica y soberana en el mundo. Se priorizan catorce sectores productivos y cinco industrias estratégicas, con el objetivo principal de producir en el Ecuador. Entre las industrias en proceso de fortalecimiento son la metalmecánica y la relacionada con la transformación de los polímeros. Buscando alinearse con el objetivo nacional, se propone el mejoramiento de un proceso de manufactura para la industria de procesamiento de polímeros. La valoración de la organización se realiza mediante la teoría de restricciones (TOC) que a través de una forma ordenada y sistemática logra el mejoramiento de un proceso productivo. Los resultados se pueden visibilizar en términos de utilidades, administrando el recurso más débil denominado restricción que existe en cualquier organización para convertirlo en una oportunidad de mejora (Abisambra, 2008); (Goldratt, 2005).

El área de diseño utiliza la herramienta CAD que consiste en el empleo de programas informáticos para de forma interactiva diseñar productos y respaldar estudios de ingeniería (Gómez, 2010). La sección de manufactura pretende migrar de un entorno de trabajo manual a un sistema CAM que contempla el uso de computadoras para dar soporte en todas las fases de manufactura de un producto (Candal, 2005).

El CAM es soportado por un sistema CNC que a través de una codificación alfanumérica permite controlar acciones y movimientos de un equipo. La implementación de un sistema CAD-CAM requiere una gran inversión por tal motivo la utilización de elementos financieros como tasa interna de retorno, análisis costo-beneficio, tiempo de retorno de la inversión y el valor actual neto permiten tomar la decisión adecuada acorde con la realidad de la industria metalmecánica-plástica (Cuatrecasas, 2009). El presente trabajo tiene como finalidad dar las directrices necesarias para poder implementar este sistema de manufactura, así como contribuir con un texto científico que sirva de guía para otras industrias con similares características a la organización evaluada (Fleitman, 2007).

\section{Materiales y métodos}

\subsection{Materiales}

Para la evaluación del proceso de manufactura y de inyección en la industria metalmecánicaplástica fue considerado un molde de acero manufacturado con seis cavidades y como materia prima polipropileno-homopolímero que se inyectó en forma de roseta como se indica en la Figura 1.

Las herramientas para la generación del molde se definieron de acuerdo con el tipo de material y maquinaria disponibles. Para el maquinado del molde se utilizó una máquina CNC 3 ejes Miltronics Controlador Centurion VI y una inyectora Van dorn 70 toneladas y 110 gramos de inyección. 


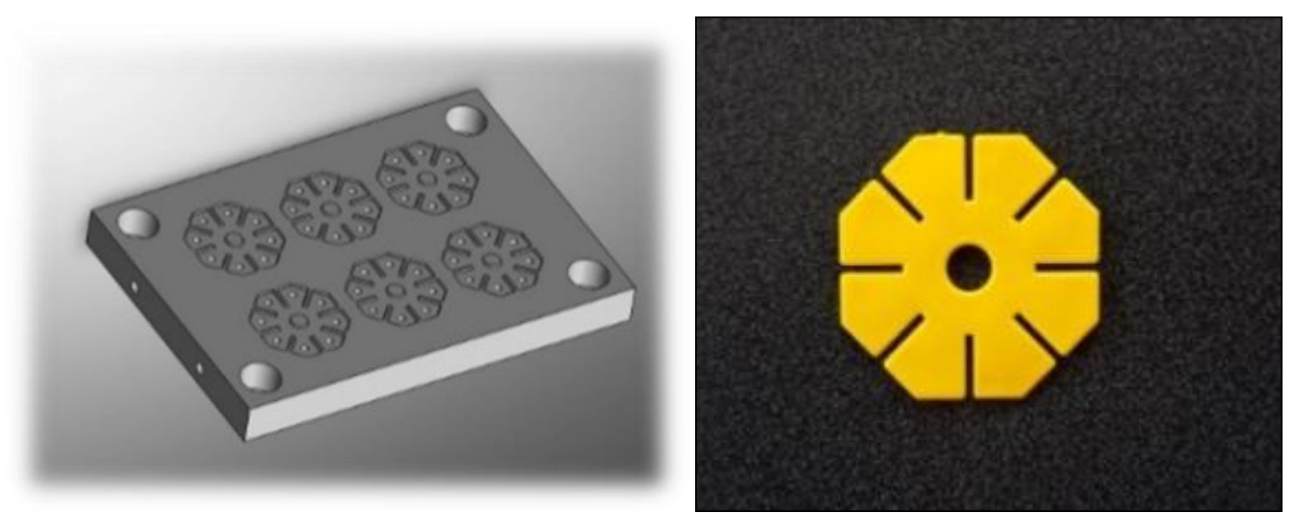

Figura 1. Roseta plástica y molde de acero de seis cavidades

\subsection{Métodos}

Para la implementación del proceso de manufactura CAD-CAM fue necesario revisar los tiempos de operación que se utilizaron durante la ejecución del proyecto, desde su diseño pasando por el maquinado del molde de acero y la inyección del polipropileno-homopolímero (Groover, 2008). Al definir los tiempos mediante la teoría de restricciones se establecieron los puntos críticos de la cadena productiva. Finalmente se valoró la alternativa más adecuada con relación al proceso anterior de manufactura.

\subsubsection{Medición del tiempo de operación}

Para cada proceso con arranque de viruta que conforma la fabricación del molde, se establecieron los tiempos de operación en función del tipo de material, tipo de herramienta disponible y clase de máquina utilizada.

El tiempo de maquinado para el torneado se define con la ecuación 1. (Kalpakjian, y otros, 2014 pág. 630)

$$
T_{m}=\frac{l}{f N}
$$

Donde:

$\begin{array}{cll}T_{m} & \text { Tiempo de maquinado } & \mathrm{min} \\ f & \text { Avance } & \mathrm{mm} / \mathrm{rev} \\ \mathrm{N} & \text { Velocidad de rotación } & \mathrm{Rpm}\end{array}$

La remoción de material en el proceso de torneado se determina con la ecuación 2. (Kalpakjian, y otros, 2014 pág. 630)

$$
M_{r r}=\pi D_{m} d f N
$$


Donde:

$\begin{array}{lll}M_{r r} & \text { Tasa de remoción de material } & \mathrm{mm}^{3} / \mathrm{min} \\ D_{m} & \text { Diámetro promedio } & \mathrm{mm} \\ d & \text { Profundidad de corte } & \mathrm{mm} \\ N & \text { Velocidad de rotación } & \mathrm{Rpm} \\ f & \text { Avance } & \mathrm{mm} / \mathrm{rev}\end{array}$

La remoción de material en el proceso de fresado se determina con la ecuación 3. (Kalpakjian, y otros, 2014 pág. 670)

$$
M_{r r}=w d v
$$

Donde:

$\begin{array}{lll}M_{r r} & \text { volumen de remoción del fresado } & \mathrm{mm}^{3} \\ w & \text { Ancho de corte } & \mathrm{mm} \\ d & \text { Profundidad de corte } & \mathrm{mm} \\ v & \text { Velocidad de avance } & \mathrm{mm} / \mathrm{min}\end{array}$

La remoción de material en el proceso de perforado se determina con la ecuación 4. (Groover, 2014 pág. 361)

$$
R_{m r}=\frac{\pi D^{2} f_{r}}{4}
$$

Donde:

$$
\begin{array}{lll}
R_{m r} & \text { Volumen de remoción del perforado } & \mathrm{mm}^{3} / \mathrm{min} \\
D & \text { Diámetro de la herramienta } & \mathrm{mm} \\
f_{r} & \text { Velocidad de avance } & \mathrm{mm} / \mathrm{min}
\end{array}
$$

\subsubsection{Aplicación de la teoría de restricciones}

La teoría de restricciones es una herramienta gerencial que permite la optimización de procesos productivos mediante la determinación de la operación que restringe el proceso en función de tiempo. En la Figura 2, se encuentran las fases que se aplicaron de la metodología de teoría de restricciones al proceso de manufactura CAD-CAM (Abisambra, 2008).

En la Figura 3, se indica el proceso de implementación de manufactura CAD-CAM con aplicación de la teoría de restricciones. 


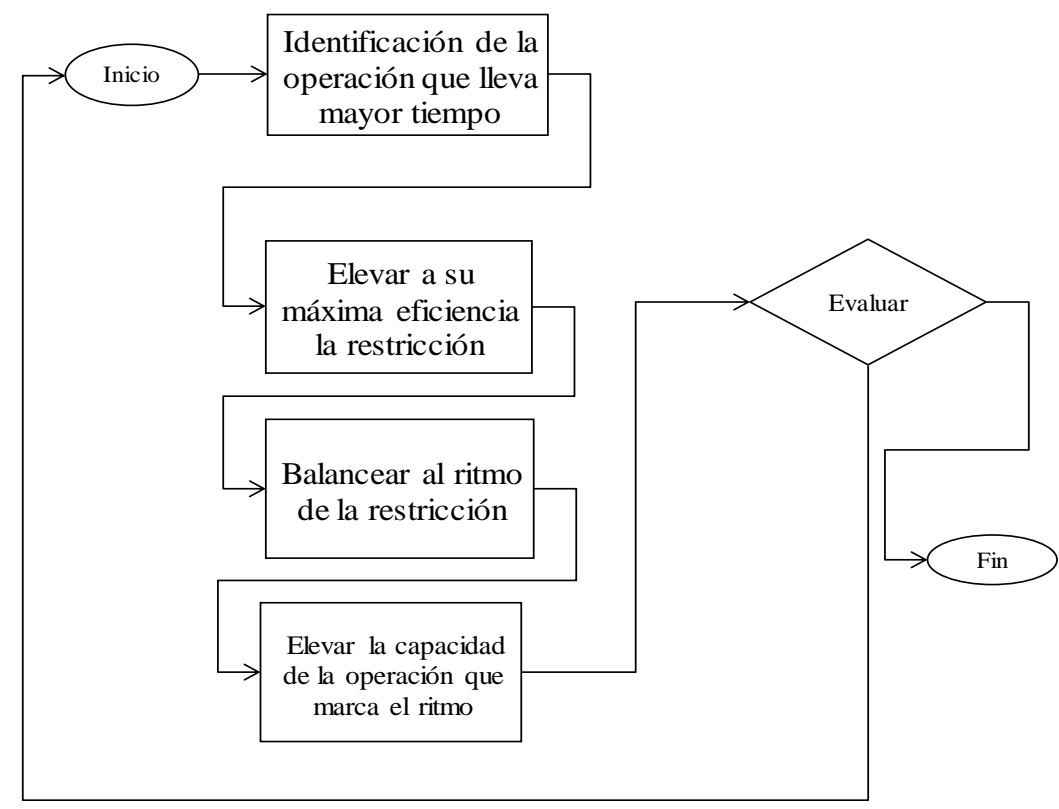

Figura 2. Aplicación de la teoría de restricciones. Fases de la metodología

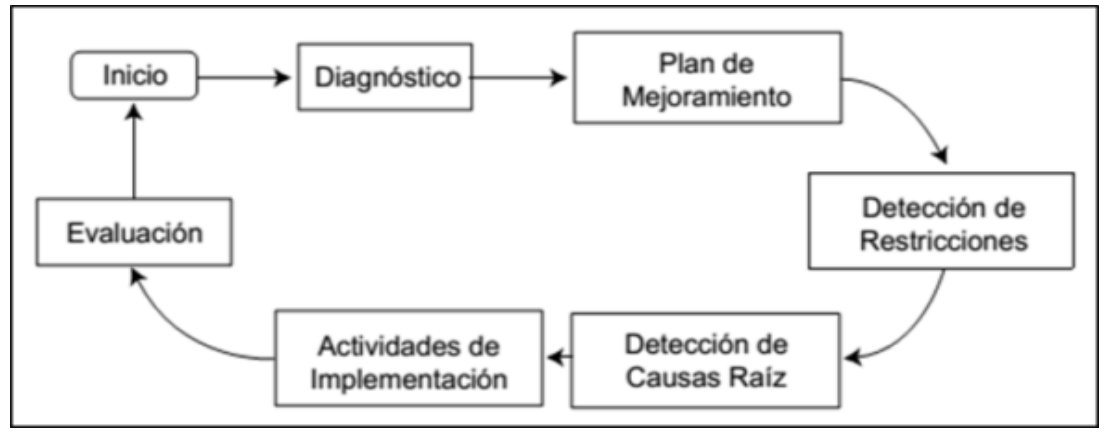

Figura 3. Implementación de manufactura CAD-CAM

\subsubsection{Criterios para la toma de decisión}

La teoría de restricciones utiliza el throughput, término que no tiene traducción definida pero se considera como la diferencia entre las ventas y la materia prima, o como la contribución marginal cuando la materia prima es el único costo variable (Abisambra, y otros, 2008).

Al definir la tecnología a implementarse, el análisis financiero es fundamental para la toma de decisión, el valor presente neto se obtiene con la ecuación 5 (Serrano Rodríguez, 2008, pág. 170).

$$
V A N=\frac{F_{n}}{(1+i)^{n}}
$$

La relación beneficio-costo se calcula como el cociente entre el valor presente de los ingresos y el valor presente de los egresos para una tasa de interés $i$, como se indica en la Ec. 6 (Serrano Rodríguez, 2008 pág. 176).

$$
\frac{B}{C}=\frac{\text { Valor presente de los ingresos } i}{\text { Valor presente de los egresos } i}
$$


Para la implementación se evaluó el tiempo de recuperación de la inversión considerando el valor presente acumulado

\section{Resultados}

\subsection{Resultado del proceso de inyección sin aplicar teoría de restricciones}

Sin aplicar teoría de restricciones, la industria metalmecánica-plástica procesa un requerimiento de 30000 unidades de roseta con un peso 8 gramos por unidad. El requerimiento total es de 240 kilogramos con un tiempo requerido de 25 horas, como se observa en la Tabla 1.

Tabla 1. Tiempo requerido de inyección de rosetas

\begin{tabular}{ll}
\hline Característica & Unidad \\
\hline Peso de roseta $(\mathrm{gr})$ & 8 \\
Requerimiento de producción (Unid) & 30000 \\
Requerimiento de producción (kg) & 240 \\
Capacidad inyectora(kg/h) & 9,6 \\
Tiempo requerido (h) & 25 \\
\hline
\end{tabular}

\subsubsection{Tiempo de diseño molde metálico forma de roseta}

Para el diseño del molde de acero se lo realizaba en 2D y no se visualizaban las zonas con formas complejas para el maquinado. La generación de simulaciones de inyección para optimizar el material y el proceso de maquinado es fundamental para el tiempo de diseño lo cual no se obtiene en modelado 2D.

\subsubsection{Tiempo de mecanizado para torneado del molde}

Para el cálculo del tiempo de torneado de cada componente del molde de acero se determinó la cantidad de material a remover y la tasa de remoción por minuto. El tiempo total para el torneado del molde en lo correspondiente a las guías es de 601,79 minutos.

\subsubsection{Tiempo de mecanizado para fresado del molde}

El proceso de fresado para las piezas que conforman el molde son de tipo lateral. El tiempo determinado para el proceso de fresado de la cavidad superior e inferior, del respaldo núcleo, porta núcleo, portacavidad, portaexpulsores, expulsor, paralela A y B es de 336,42. 


\subsubsection{Tiempo de mecanizado para taladro del molde}

Para el tiempo total de perforado se consideraron la tasa de remoción de material, el volumen de material por eliminar y la secuencia de taladrado con diferentes diámetros de broca. El tiempo de mecanizado del taladrado de la cavidad superior e inferior, respaldo macho, porta-emulsores, expulsor, portacavidad y portamacho es de 257,09 minutos.

\subsubsection{Tiempo de servicio contratado de mecanizado}

Al no contar con un sistema que permita generar maquinados con alta precisión, exactitud de medidas y formas, se contrata el servicio de CNC para la fabricación de las cavidades superiores e inferiores del molde. El tiempo requerido para la fabricación de las cavidades superiores e inferiores para la inyección de roseta por el proveedor es de 120 horas con un costo de 4800,00 USD (Juiña, 2015).

En la Figura 4, se indica la distribución de tiempos para el desarrollo del proyecto de la pieza roseta de polipropileno-homopolímero. El proceso de Diseño corresponde al 27\%, el de mecanizado del molde con sus procesos de torneado $5 \%$, fresado $2 \%$ y perforado $2 \%$. El tiempo de producción en lo referente a la inyección para obtener la pieza en forma de roseta corresponde al $11 \%$ y el maquinado de la forma de roseta en el molde de acero corresponde al 53\%.

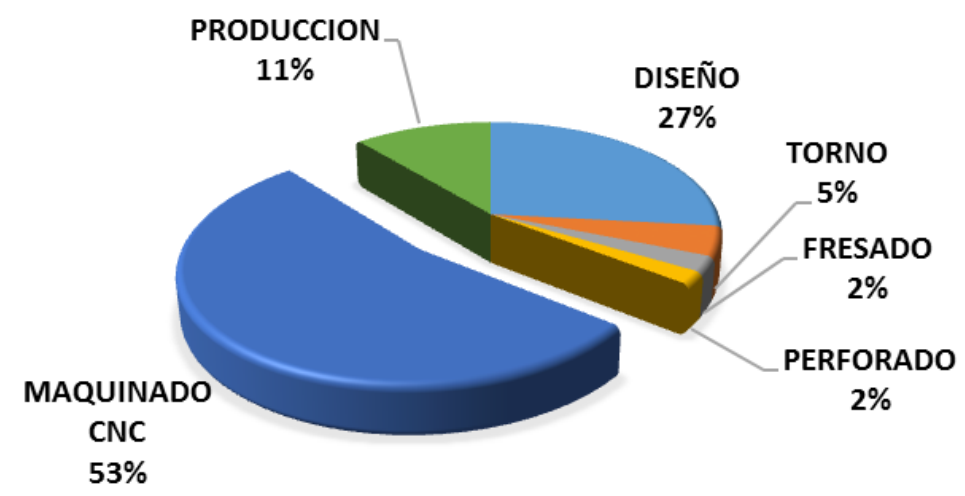

Figura 4. Distribución de tiempos para el proyecto roseta plástica

Para la aplicación de la teoría de restricciones en la industria metalmecánica-plástica fue necesario determinar los tiempos de cada uno de los procesos con lo que se llevaba a cabo el proyecto de la roseta de polipropileno-homopolímero.

\subsection{Identificación de la restricción aplicando TOC}

En la Tabla 2, se detallan los resultados de la evaluación tanto en tiempo como en costos operativos, considerando la situación actual de la organización. 
Tabla 2. Tiempos y costos actuales

\begin{tabular}{lrrr}
\hline \multirow{2}{*}{ OPERACIÓN } & TIEMPO $(\mathbf{h})$ & COSTO POR HORA (USD/h) & COSTO OPERACIÓN (USD) \\
\hline DISEÑO & 60,00 & 10 & $\mathbf{6 0 0 , 0 0}$ \\
TORNO & 10,03 & 12 & $\mathbf{1 2 0 , 3 6}$ \\
FRESADO & 5,23 & 10 & $\mathbf{5 2 , 3 0}$ \\
PERFORADO & 4,3 & 8 & $\mathbf{3 4 , 4 0}$ \\
SERVICIO CNC & 120,00 & & $\mathbf{4 . 5 0 0 , 0 0}$ \\
PRODUCCIÓN & 25,00 & 10 & $\mathbf{2 5 0 , 0 0}$ \\
\hline TIEMPO TOTAL & $\mathbf{2 2 4 , 5 6}$ & COSTO TOTAL & $\mathbf{5 . 5 5 7 , 0 6}$ \\
\hline
\end{tabular}

El porcentaje del servicio de mecanizado es del 53\% y de diseño con $27 \%$ del tiempo para el desarrollo de la roseta. Mediante un diagrama de Pareto, que se indica en la fig. 5 , el servicio de mencanizado CNC y la operación de diseño toman el 80\% del tiempo total de proceso, impactando directamente en el tiempo del proyecto para la obtención de la roseta didáctica en polipropilenohomopolímero. Las restricciones del mecanizado CNC se debe a la falta de un equipo propio para la manufactura asistida por computador. La restricción en el diseño es por el software utilizado, que permite el desarrollo en 2D, lo que limita la simulación del proceso de inyección.

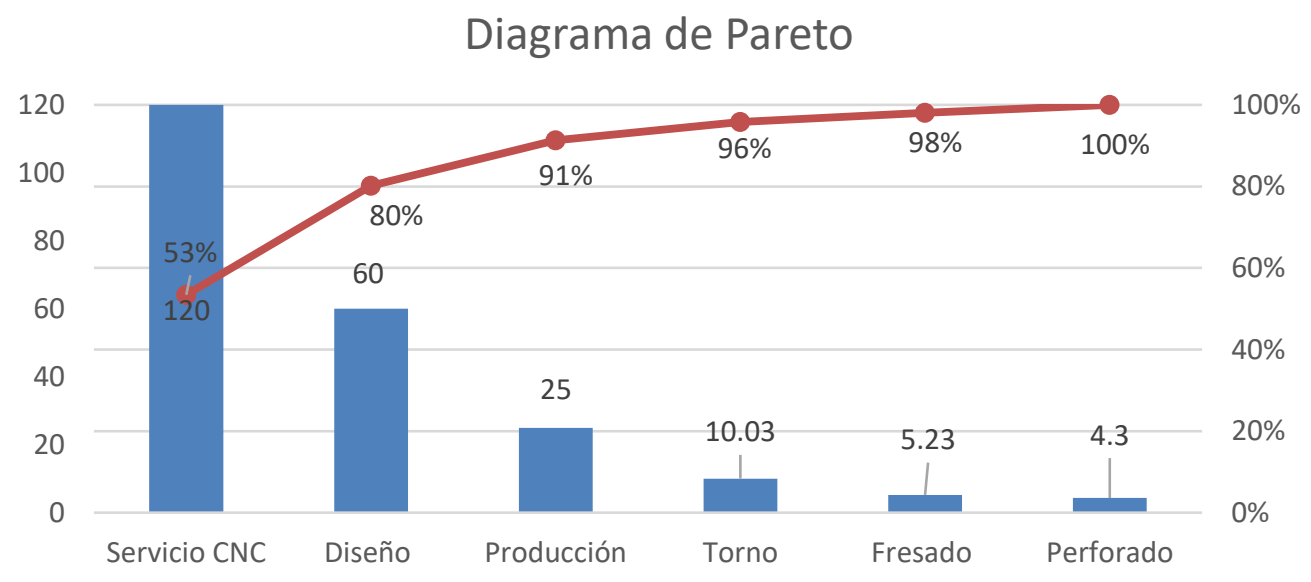

Figura 5. Diagrama de Pareto

\subsubsection{Máxima eficiencia de la restricción}

Para el proceso de mecanizado CNC se contrató dos empresas para mejorar la eficiencia en el servicio; sin embargo, se presentaron problemas de calidad y ensamble. Razón por la cual el servicio contratado es con una solo empresa. Para maximizar el área de diseño se procede a asignar una persona adicional que permita mejorar el rendimiento de generación de planos; sin embargo, la restricción en el aspecto computacional se mantiene.

\subsubsection{Elevar la capacidad de la restricción}

Al identificar las restricciones del mecanizado como del diseño, se procede a emigrar de un entorno 2-D al modelado 3-D y simulación de modelos matemáticos. En lo que respecta al mecanizado 
queda en evidencia crear lazos entre el modelado con la fabricación, que corresponde al sistema CAD-CAM.

\subsection{Resultado de la valoración del sistema CAD CAM}

Para la valoración del sistema CAD CAM se utiliza el método de factores ponderados, que permite introducir objetividad y factores que reflejan la importancia relativa de cada aspecto considerado, (Heizer , y otros, 2007 pág. 403), en la Tabla 3, se indica el criterio y la ponderación asignada.

Tabla 3. Factores de ponderación.

\begin{tabular}{cc}
\hline Valoración & Ponderación \\
\hline Alto-excelente & 5 \\
Medio-normal & 3 \\
Bajo-deficiente & 0
\end{tabular}

\subsubsection{Valoración software CAD}

El software CAD es evaluado de acuerdo con los siguientes aspectos:

Capacidad técnica del programa; en la Tabla 4, se describen los criterios emitidos y la puntuación obtenida.

Tabla 4. Capacidad técnica.

\begin{tabular}{lcccc}
\hline & AutoCAD & Solidworks & Inventor & Valor ideal \\
\hline Modelo paramétrico & 0 & 5 & 5 & 5 \\
Manejo de superficies & 0 & 5 & 5 & 5 \\
Ensamble & 3 & 5 & 5 & 5 \\
Planos & 5 & 5 & 5 & 5 \\
Simulación estática dinámica & 0 & 5 & 5 & 5 \\
Simulación de inyección plástico & 0 & 5 & 0 & 5 \\
Total & $\mathbf{7}$ & $\mathbf{3 0}$ & $\mathbf{2 5}$ & $\mathbf{3 0}$ \\
\hline Porcentaje & $\mathbf{2 3 . 3 \%}$ & $\mathbf{1 0 0 \%}$ & $83,3 \%$ & $\mathbf{1 0 0 \%}$ \\
\hline
\end{tabular}

Disponibilidad, son criterios relacionados con el servicio de los proveedores locales de software especializados para manufactura, en la Tabla 5 se describen los valores obtenidos.

Tabla 5. Disponibilidad

\begin{tabular}{lcccc}
\hline & AutoCAD & Solidworks & Inventor & Valor idea \\
\hline Servicio de venta & 5 & 5 & 5 & 5 \\
Capacitación & 0 & 5 & 0 & 5 \\
Actualización & 0 & 5 & 0 & 5 \\
Total & $\mathbf{5}$ & $\mathbf{1 5}$ & $\mathbf{5}$ & $\mathbf{1 5}$ \\
\hline Porcentaje & $33,3 \%$ & $\mathbf{1 0 0} \%$ & $33,3 \%$ & $\mathbf{1 0 0 \%}$ \\
\hline
\end{tabular}

En la Tabla 6 se expresa la matriz final de resultados para la selección del software CAD. 
Tabla 6. Matriz final

\begin{tabular}{lcccc}
\hline & AutoCAD & Solidworks & Inventor & Valor ideal \\
\hline Capacidad & $23,3 \%$ & $100 \%$ & $83,3 \%$ & $100 \%$ \\
Disponibilidad & $33,3 \%$ & $100 \%$ & $33,3 \%$ & $100 \%$ \\
\hline Total & $28,3 \%$ & $\mathbf{1 0 0} \%$ & $58,3 \%$ & $\mathbf{1 0 0} \%$ \\
\hline
\end{tabular}

Con los resultados obtenidos, el software Solidworks se ajusta a las necesidades de la organización, aun cuando su precio es de 25.750 Usd, y representa 5,7 veces más que la opción 2 de evaluación.

\subsubsection{Valoración del software CAM}

El programa de manufactura permite integrar el modelo 3D generado por el CAD, a la máquina de control numérico computarizado. Para la evaluación del sistema CAM se considera los siguientes aspectos:

Aspectos técnicos, en la Tabla 7, se detallan los criterios evaluados para los diferentes software que se ofrecen en el mercado local.

Tabla 7. Aspectos técnicos

\begin{tabular}{lcccc}
\hline & MASTERCAM & SolidEdge expresscam & SOLIDCAM & Valor ideal \\
\hline Manufactura 4 ejes & 0 & 0 & 0 & 5 \\
Manufactura 3 ejes & 5 & 5 & 5 & 5 \\
Manufactura torno & 5 & 5 & 5 & 5 \\
Manufactura erosión & 3 & 3 & 0 & 5 \\
Entorno único con & 0 & 0 & 5 & 5 \\
programa CAD & $\mathbf{1 3}$ & $\mathbf{1 3}$ & $\mathbf{1 5}$ & $\mathbf{2 5}$ \\
Total & $52 \%$ & $52 \%$ & $\mathbf{6 0 \%}$ & $\mathbf{1 0 0 \%}$ \\
\hline Porcentaje & & & & \\
\hline
\end{tabular}

Disponibilidad, en la Tabla 8 se indica la ponderación para la disponibilidad del programa a nivel local

Tabla 8. Disponibilidad

\begin{tabular}{lcccc}
\hline & MasterCam & SolidEdge exprescam & SOLIDCAM & VALOR IDEAL \\
\hline Servicio de venta & 5 & 5 & 5 & 5 \\
Capacitación & 0 & 0 & 5 & 5 \\
Actualización & 0 & 5 & 0 & 5 \\
Total & $\mathbf{5}$ & $\mathbf{1 0}$ & $\mathbf{5}$ & $\mathbf{1 5}$ \\
\hline Porcentaje & $33,3 \%$ & $\mathbf{6 6 , 6 \%}$ & $\mathbf{6 6 , 6 \%}$ & $\mathbf{1 0 0 \%}$ \\
\hline
\end{tabular}

En la Tabla 9 se expresa la matriz final de resultados para la selección del software CAM.

Tabla 9. Matriz final software CAM

\begin{tabular}{lcccc}
\hline & Mastercam & Solid Edge & Solidcam & Valor ideal \\
\hline Aspecto técnico & $52 \%$ & $52 \%$ & $60 \%$ & $100 \%$ \\
Disponibilidad & $33,3 \%$ & $66,6 \%$ & $66,6 \%$ & $100 \%$ \\
\hline Total & $46,5 \%$ & $59,3 \%$ & $\mathbf{6 3 , 3} \%$ & $\mathbf{1 0 0} \%$ \\
\hline
\end{tabular}




\subsubsection{Valoración equipo CNC}

Para complementar el sistema CAD-CAM fue necesario la adquisición de un equipo CNC, los factores que se evaluaron, fueron: aspectos tecnológicos, aspectos informáticos y costo. El equipo CNC Romi D 1000 con el valor ponderado del 99\%, la otra alternativa fue el equipo HASS VF3 con el $92 \%$, Milltronics MB20 con el $55 \%$ y el equipo C-TEK con el $80 \%$. A pesar que el equipo CNC Romi D 1000 cumple con el mejor valor ponderado, el costo de la inversión será el más importante para la toma de decisión. El equipo que se considerará para la implementación es la máquina Milltronics MB20 con la inversión de 25.600 USD, con relación al equipo Romi D 1.000 de inversión 10.0796,9 USD, el HASS VF3 con 99.950 USD de inversión y el C-TEK con 54.500 USD.

\subsection{Resultados de la implementación}

El sistema CAD seleccionado es el programa SolidWorks conjuntamente con el sistema SolidCAM por ser una plataforma única que evita la pérdida de información durante el traspaso de información entre las dos plataformas. El equipo seleccionado es la máquina Milltronics MB 20 que se indica en la Figura 6.

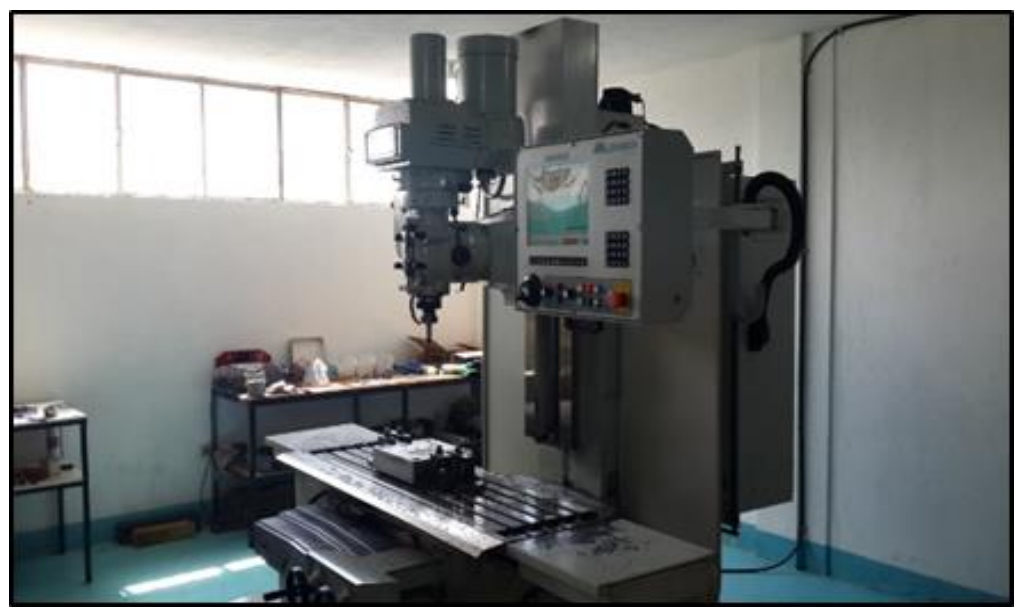

Figura 6. Máquina Milltronics MB20

\subsubsection{Evaluación sistema CAD}

El sistema CAD se denomina SolidWorks, las ventajas que ofrece es el modelado en 3 dimensiones, manejo de entornos de trabajo como pieza, ensamble, plano. Adicionalmente, permite la evaluación de los modelos con análisis estáticos y dinámicos. Para el diseño de elementos plásticos tiene un módulo que facilita la ingeniería aplicada al proceso de polímeros. En la Figura 7 se detalla la simulación de inyección de la pieza de polipropileno-homopolímero, para identificar las zonas donde se generan temperaturas y vaciados críticos durante su proceso.

Con el sistema CAD implementado correspondiente al SolidWorks el tiempo total para el diseño es de 12,63 horas, como se observa en la Tabla 10. 


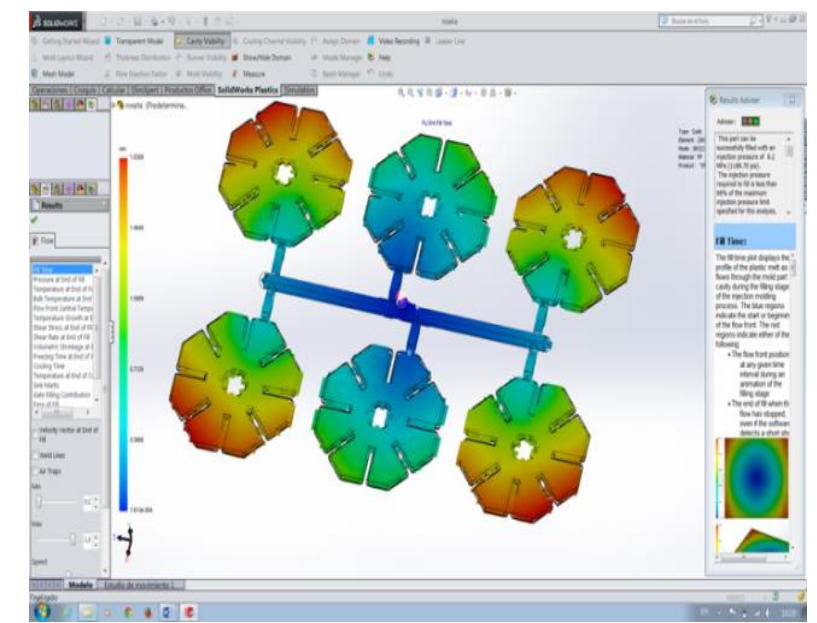

Figura 7. Simulación de inyección plástica

Tabla 10. Tiempo total para el diseño

\begin{tabular}{lcccc}
\hline \multicolumn{1}{c}{ Elemento } & $\begin{array}{c}\text { Diseño } \\
(\mathbf{m i n})\end{array}$ & $\begin{array}{c}\text { Simulación } \\
(\mathbf{m i n})\end{array}$ & $\begin{array}{c}\text { Plano } \\
(\mathbf{m i n})\end{array}$ & $\begin{array}{c}\text { Total } \\
(\mathbf{m i n})\end{array}$ \\
\hline Roseta & 30 & 8 & 10 & 48 \\
Cavidad superior & 40 & 10 & 30 & 80 \\
Cavidad inferior & 60 & 10 & 45 & 115 \\
Porta cavidad & 40 & 0 & 30 & 70 \\
Porta núcleo & 40 & 0 & 30 & 70 \\
Respaldo núcleo & 30 & 0 & 20 & 50 \\
Placa portapropulsores & 20 & 0 & 20 & 40 \\
Placa expulsora & 15 & 0 & 15 & 30 \\
Expulsores & 5 & 0 & 5 & 10 \\
Guías & 15 & 0 & 15 & 30 \\
Perno guía & 15 & 0 & 15 & 30 \\
Paralela & 10 & 0 & 10 & 20 \\
Ensamble & 75 & 0 & 90 & 165 \\
\hline Total (min) & & & & 758 \\
Total (h) & & & & 12,63 \\
\hline
\end{tabular}

\subsubsection{Evaluación sistema CAM}

La evaluación del sistema CAM contiene el desarrollo del modelo CAM con referencia a la máquina adquirida. Para el proyecto de roseta plástica que se indica en la Figura 8, se evaluó el proceso de maquinado de las cavidades conjuntamente con el sistema de guías.

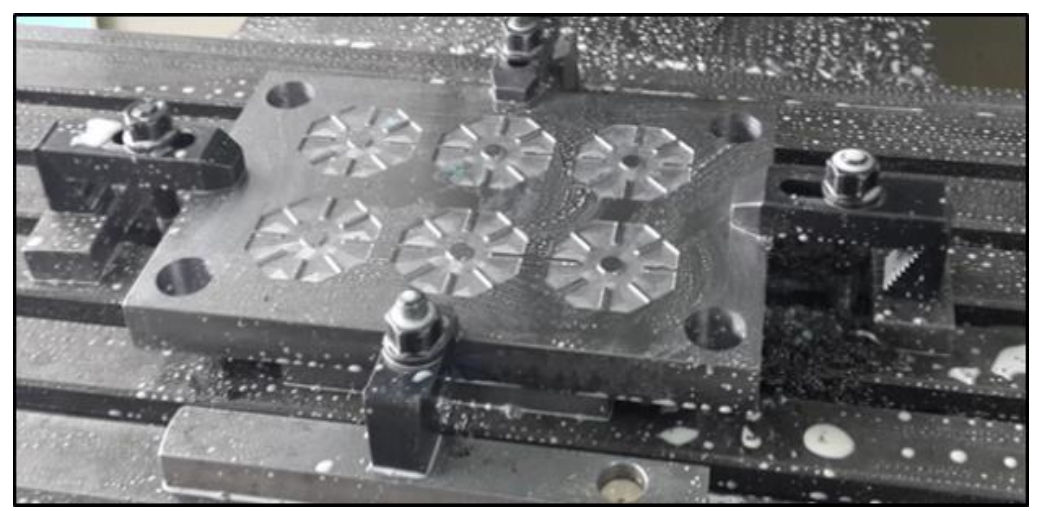

Figura 8. Maquinado de cavidades

El tiempo total para el mecanizado de la cavidad superior e inferior corresponde a 14,71 horas. 


\subsection{Resultados de la implementación CAD-CAM}

En la Tabla 11, se describen los resultados finales luego de la implementación del sistema CAD CAM, en la empresa.

Tabla 11. Resultado CAD CAM

\begin{tabular}{ccccc}
\hline Área & Tiempo anterior $(\mathbf{h})$ & Tiempo actual $(\mathbf{h})$ & Reducción & Porcentaje de mejora (\%) \\
\hline Diseño & 60 & 12,63 & 47,37 & $\mathbf{7 9 \%}$ \\
CAM & 120 & 14.71 & 105,29 & $\mathbf{8 8 \%}$
\end{tabular}

En la Figura 9 se indican los resultados globales de la implementación del sistema CAD CAM en la organización.

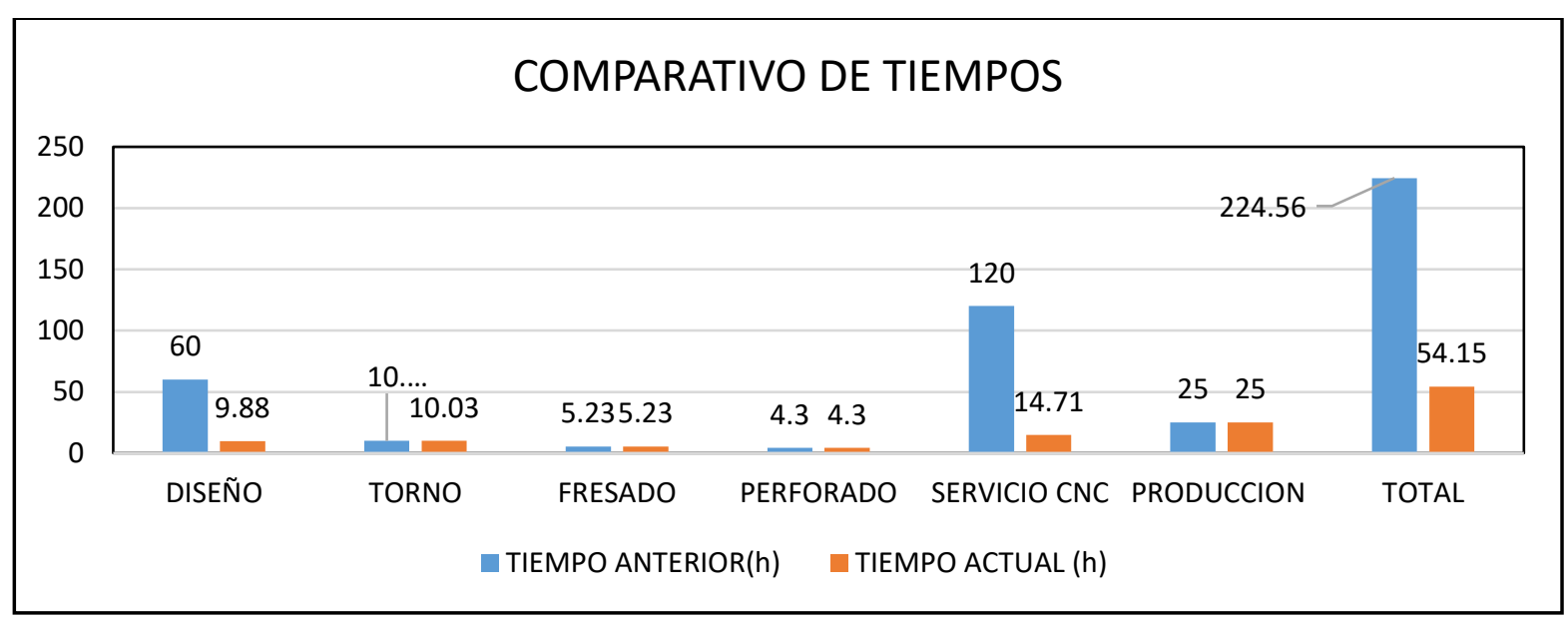

Figura 9. Comparativo de tiempos

Adicionalmente se determinaron nuevos tiempos y distancias relacionadas con los traslados que se expresan en la Tabla 12.

Tabla 12. Resultados generales de traslados y tiempos

\begin{tabular}{lrrr}
\hline \multicolumn{4}{c}{ TIEMPO (h) } \\
\hline Operaciones & $5.635,3$ & $3.675,72$ & TIEMPO (\%) \\
Transporte & 225 & 165 & $34,70 \%$ \\
Controles & 330 & 320 & $36 \%$ \\
Esperas & 7.200 & 0 & Eliminación 100\% \\
Almacenamiento & 0 & 0 & \\
TOTAL & $\mathbf{1 3 . 3 9 0 , 3}$ & $\mathbf{4 . 1 6 0 , 7 2}$ & Reducción 69\% \\
\hline \multicolumn{4}{c}{ DISTANCIA (m) } \\
\hline Operaciones & ANTERIOR & $\mathbf{C N C}$ & RECORRIDO (\%) \\
Transporte & 33 & 34 & AUMENTO 3\% \\
Controles & 47.630 & 5.030 & REDUCCIÓN 89\% \\
Esperas & 4 & 5 & AUMENTO 25\% \\
Almacenamiento & & & \\
TOTAL & $\mathbf{4 7 . 6 6 7}$ & $\mathbf{5 . 0 6 9}$ & Reducción 89,40\%
\end{tabular}




\subsection{Análisis financiero}

Para la implementación del Sistema CAD CAM se realizaron egresos de dinero que se expresan en la Tabla 13.

Tabla 13. Egreso de dinero para la implementación CAD CAM

\begin{tabular}{lccccc}
\hline & Valor (USD) & Interes (\%) & Periodo (meses) & Pago final (USD) & Pago mensual \\
\hline Efectivo & 10710,69 & $15,2 \%$ & 36 & $13.420,40$ & 372,34 \\
Préstamo & 14889,33 & $15,2 \%$ & 36 & $19.230,12$ & 534,17 \\
Pago mensual & & & & & $\mathbf{9 0 6 , 5 1}$
\end{tabular}

La tasa de interés de oportunidad se consideró de 15,90 \% como valor de consumo, con el desarrollo del flujo de caja dentro de la organización, se obtuvieron los siguientes resultados: en el período 7 se generó un VAN positivo de 365,27 USD., valor TIR 16,2\%, el beneficio costo es de 1,01, y la aproximación del tiempo de retorno de la inversión es de 6,94 años.

\subsubsection{Throughput}

En la Tabla 14, se expresan los valores comparativos de la contribución marginal para el proceso de rosetas plásticas.

Tabla 14. Análisis comparativo throughput

\begin{tabular}{lccccc}
\cline { 2 - 6 } & $\begin{array}{l}\text { VENTA } \\
\text { MOLDE }\end{array}$ & $\begin{array}{c}\text { COSTO } \\
\text { MATERIALES }\end{array}$ & $\begin{array}{c}\text { COSTO } \\
\text { MAQUINADO }\end{array}$ & $\begin{array}{c}\text { COSTO } \\
\text { CNC }\end{array}$ & MARGEN \\
\cline { 2 - 6 } Anterior & $8.450,00$ & $2.400,00$ & $1,057,06$ & $4.500,00$ & 492,94 \\
Mejora & $8.450,00$ & $2.400,00$ & $1.057,06$ & $1.298,39$ & $3.694,55$
\end{tabular}

\section{Conclusiones y recomendaciones}

La de la teoría de restricciones puede aplicarse en la industria que utiliza modelado y simulación. Es una herramienta para evaluar inicialmente las oportunidades de mejora e implementarlas. En el presente proyecto la problemática fue identificada en sistema CAD-CAM, en la cual se mencionan restricciones referentes al diseño de la roseta y mecanizado del molde de acero.

En las industrias que realizan diseño, desarrollo y mejoramiento continuo de productos es fundamental utilizar un sistema que relacione el modelo a desarrollar y el comportamiento en su manufactura, como fue el caso de la roseta que al implementar el sistema CAD-CAM las operaciones de diseño se las efectúa en 9,88 horas y el proceso CNC en 14,71 horas con un porcentaje de reducción correspondiente al $69 \%$ del tiempo total del proyecto.

Al eliminar la concesión del servicio de CAM se redujo el tiempo en 105,29 horas y se logró descartar el tiempo de espera y se redujo la distancia de traslado en un $89,4 \%$.

Se debe considerar que al aplicar la teoría de restricciones en la industria, la restricción se traslada a otro proceso como se observa en la implementación del sistema CAD-CAM. Se adquirió un equipo CNC que eliminó el servicio externo de mecanizado, que ocasionó el traslado de la restricción al 
área comercial, lo cual implica gestión de nuevos clientes para ofrecer el servicio de mecanizado de moldes de acero en distintas industrias del sector plástico.

Con la implementación del sistema de Manufactura CAD CAM, el throughput de la organización aumentó a 3.694,55 USD; sin embargo, es necesario indicar que en cada proyecto relacionado con moldes para plástico este indicador podrá variar.

El desarrollo de nuevos productos permite al modelamiento y a la simulación interaccionar entre sí, como es el caso de la implementación del programa de manufactura que permite integrar el modelo 3D generado por el CAD con el equipo de control numérico computarizado para la fabricación del molde de acero. Con la implementación del sistema CAD-CAM se puede observar en 3D el proyecto roseta y el molde de acero final, lo cual permite simular el proceso de inyección del polipropileno material constitutivo de la roseta con relación al molde.

\section{Bibliografía}

Abisambra L. (2008). Aplicación de la Teoría de Restricciones a los Procesos de Producción de la Planta de Fundición de Imusa. Soluciones de Posgrado EIA, pp. 121-133.

Bodini G. (1992). Moldes y Máquinas de Inyección para la Transformación de Plásticos. México, McGraw-Hill.

Candal M. (2005). Integración CAD/CAE/CAM-PR en la Optimización del Diseño de Productos Plásticos. Ciencia e Ingeniería.

Cuatrecasas L. (2009). Diseño Avanzado de Procesos y Plantas de Producción Flexible, Barcelona, Bresca.

Fleitman J. (2007). Evaluación Integral para Implantar Modelos de Calidad. México, Pax México.

Goldratt E. (2005). La Meta, Madrid, Edigrafos.

Gómez S. (2010). SolidWorks. México. Alfaomega.

Groover M. (2008). Automation, Production Systems and Computer-Integrated Manufacturing. New Jersey. Prentice Hall.

Manual del Mecánico Industrial, Máquinas y Control Numérico. (2000). Madrid, Cultural.

Heizer J. (2010). Dirección de la Producción y Operaciones. Madrid. Pearson Education.

Juiña L. (2015). Implementación de un proceso de Sistema de Manufactura CAD-CAM en la sección matricería de la empresa COBIPLAST. MSc. tesis, Facultad de Ingeniería Química y Agroindustria, EPN., Quito, Ecuador. 
Kalpakjian S. (2008). Manufactura, Ingeniería y Tecnología. México. Pearson.

Krajewski L. (2008). Administración de Operaciones. México, Pearson.

Niebel B. (2004). Ingeniería Industrial, Métodos, Estándares y Diseño del Trabajo. México. Alfaomega.

Richardson T. (2003). Industria del Plástico. Madrid. Paraninfo.

Sánchez S. (2001). Moldeo por inyección de termoplásticos. México, Limusa.

Tompkins J. (2011). Planeación de Instalaciones. México, Cengage Learning.

Serrano Rodriguez, Javier. 2008. Matemáticas Financieras . Bogota : Alfaomega, 2008. 